\title{
Hydrodynamic Lubrication of Textured Journal Bearing Considering Slippage: Two-dimensional CFD Analysis Using Multiphase Cavitation Model
}

\author{
M. Tauviqirrahmana, A. Pratamaa, Jamaria, Muchammad ${ }^{b}$ \\ a Laboratory for Engineering Design and Tribology, Department of Mechanical Engineering, Diponegoro University, \\ Jl. Prof. Soedharto, SH., Semarang, Central Java 50275, Indonesia. \\ ${ }^{\mathrm{b}}$ Laboratory for Surface Technology and Tribology, Faculty of Engineering Technology, Twente University, \\ Drienerloolan 5, Postbus 217, 7500 AE, Enschede, The Netherlands.
}

Keywords:

\section{Cavitation}

Computational fluid dynamics (CFD)

Slippage

Texturing

\section{Corresponding author:}

Mohammad Tauviqirrahman Laboratory for Engineering Design and Tribology, Department of Mechanical Engineering,

Diponegoro University, Jl. Prof. Soedharto, SH, Semarang, Central Java 50275, Indonesia.

E-mail:

mtauviq99@lecturer.undip.ac.id

\begin{abstract}
A B S T R A C T
Partial texturing of the surface of journal bearings have been proven very beneficial in terms of friction coefficient. In the present work, the load support of the hydrodynamic textured journal bearing combined with artificial slippage is fully characterized by means of computational fluid dynamics (CFD) simulations based on the numerical solution of the Navier-Stokes equations for incompressible flow. In order to model slippage, the enhanced user-defined-function (UDF) code is developed. Realistic boundary condition is employed by implementing the mixture multiphase model to model a cavitation in the bearing. The numerical analysis is performed under the condition of different groove depths, eccentricity ratios and slippage placements along the textured area of bearing. The simulation results including hydrodynamic pressure and load support are gained and compared for conventional smooth parameters. A reference to determine optimal groove depths as well as best artificial slippage placement of textured bearing under different conditions of loading are proposed. Based on the present results, favorable slippagetextured journal bearing design can be assessed.
\end{abstract}

(C) 2019 Published by Faculty of Engineering

\section{INTRODUCTION}

With increasing worldwide demand for more efficient energy consumption, the use of textured surface has emerged out intensive attentions of tribologist and lubrication scientist all over the world. A positive influence of textured surface on the performance of lubricated contact in reducing the friction and increasing the additional load support is highlighted. During the past decades, due to these attractive features of surface texturing, the researchers have focused their work effort to explore the effect of textured surface on the journal bearing experimentally (see for example [1,2]) and/or numerically [3-6]. Up to now, finding optimal texturing parameters is still very challenging due to the lack of universal design recommendations for textured journal bearing. 
Recently, to handle more complex problems of textured journal bearing and with the development of computer technology, many workers have implemented commercial CFD software which are based on full Navier-Stokes equation. The reason behind this is that the Navier-Stokes equation can revoke limitations under the situations with clear inertia effect of the lubricant. However, according to literature survey by Gropper et al. [7], a number of workers contributed to today's study around surface texturing using CFD are very limited. One of the earliest researches emphasizing the use of CFD on texturing discussion was conducted by Cupillard et al. [4]. They studied partially textured journal bearings and recommended the choice of texture depth for different eccentricity ratios. Meng et al. [5] made the comparison of tribological performance between journal bearing with compound dimple and that with simple dimple. The authors highlighted that the compound dimple has a high efficiency in reducing the friction coefficient and providing the additional load support of the journal bearing. In recent publication, Lin at al. [6] pointed out that the surface texture may improve or deteriorate the performance of the journal bearing depending on the texture position in circumferential direction.

It is noteworthy that the aforementioned studies assumed the contacting surfaces as a no-slip condition. As widely known, in classical lubrication theory, the so-called "no-slippage condition" is often used, namely, the velocity at the fluid-solid interface is equal to zero. This boundary condition is the universally accepted approximation only under macroscopic sense. With the advent of new experimental technology to measure the microscopic-scale, the partial slippage has been observed on the fluid-solid interface [8]. In recent years, with the development of surface engineering, the slippage at a fluid-solid interface induced by (super)hydrophobic surface can be engineered to enhance the performance of lubricated contact. By introducing the slippage at the boundaries, a lower pressure is required to move the same amount of lubricant, and thus the reduced energy is achieved. It is worth to mention the early theoretical work of Spikes [9] who proposed the design of the bearing whose ability to not be fully wetted by a fluid by introducing the slippage. His studies on bearings with different configurations found that very low friction was reported when compared with traditional bearings. Afterwards, some workers have evaluated the application of the artificial slippage (i.e. heterogeneous slip/no-slip surface) on hydrodynamic bearings. For instance, Fortier and Salant [10] reported that artificial slippage surface in journal bearing leads to the increased load support and the reduced friction. Ma et al. [11] revealed that the optimization of the size and the shape of the slip area provides the journal bearing many advanced behaviors. An identical result was shown by Wang et al. [12]. A large pressure, load support and end leakage rate but low friction were recorded when artificial slippage was employed. Additionally, to enhance the stability performance of the threelayered journal bearing, Rao et al. [13] suggested the use of partial slip on bearing surface by choosing the appropriate eccentricity ratio and optimal angular extent of partial slip region.

Generally speaking, the evidences mentioned earlier suggest that in order to achieve a higher load support but lower friction coefficient, the use of slippage surface as well as textured surface in journal bearing is advisable. But is it possible to combine the effect of texturing and slippage? In the last few years, many works have been dedicated to the study of combined effects of texturing and artificial slippage; the conclusion was that those effects were found to be critical hypotheses to enhance the behavior of the journal bearing performance. Rao et al. [14] evaluated the performance of partially slip textured concentric journal bearing in gaining the additional load support and reducing the friction force. Aurelian et al. [15] revealed that in combined partial texturing and artificial slippage pattern, a larger reduction in power loss is highlighted as compared to the simple partial texturing. Tauviqirrahman and his group [1618] found that the combined texture/slip configuration, even in the parallel gap situation, when having low texture cell aspect ratio, high slip length and low critical shear stress condition gives higher load support but lower friction force. Ismail and Sarangi [19] numerically investigated the influence of slippage and the texture shape on the performance of parallel sliding lubricated contact. The authors found that compared to the traditional textured contact, introducing the artificial slippage around the surface texture could improve the 
performance textured parallel sliding surface. Later, using CFD method Lin et al. [20] conducted a numerical study on the influences of combined slip and surface texture focusing on the optimization of the surface design of journal bearing. The effect of employing a slip/texture area to only parts of bearing was of particular interest. Their main finding was that the enhanced tribological performance of journal bearing could be achieved with well-designed slip/texture surface. In recent lubrication, using numerical model based on complementary variables, Biancofiore et al. [21] reported that a very large increase in the pressure profile and significant decrease in friction coefficient are registered by introducing slippage.

In general, based on literature survey the textured surface combined with artificial slippage is an effective means of controlling lubrication performance in journal bearing. However, to date, based on the available literature surprisingly numerous textured bearing investigations appear to have used Reynolds approach in their work in particular while incorporating the slippage effect in journal bearing. The application of Reynolds equation in textured contacts analysis is questionable when the contacts are operating at moderate to high Reynolds number as often found in journal bearing [22]. In addition, according to Dobrica and Fillon [23], for cases having low values of textured aspect ratio as used in the present study, employing the Reynolds theory may not be appropriate. Additionally, it is noted that up until now, almost all the studies on the lubrication problem of journal bearing are limited to single-phase flow analysis. As widely known, in journal bearing under the cavitation condition the gas-liquid mixture flow exists as highlighted by recent workers [24-26]. Therefore, more work is required in the area of textured journal bearing considering slippage and multiphase cavitation.

In order to represent more real condition, in this research a multiphase flow analysis is employed to model the cavitation phenomena which allows the phase change of lubricant. The lubricated journal bearing performance is calculated by solving the Navier-Stokes for representative values of eccentricity ratio. As a note, by employing the Navier-Stokes equation the inertia terms, which is often neglected in previously published works, can be solved. This present work is of direct significance for the application and the promotion of the artificial slippage, in particular regarding the effect of surface texturing. Based on the CFD-based modeling this paper aims to elucidate the potential of surface texturing combined with artificial slippage applied to journal bearing.

This paper is divided into four sections. Firstly, a background of the research is presented. The second point explores the governing equations and numerical approach, followed by the analysis of different purely textured bearing configurations. Subsequently in third part, the promotion of the slippage on textured surface varying its placement is studied. In some cases, such performance will be compared with the performance of a conventional smooth (without textured) sliding contact. Finally, in the last section the main interesting results are concluded.

\section{METHOD}

\subsection{Governing equations of continuum mechanics}

In the present study, the lubricant flow induced by the wall motion is calculated by solving the Navier-Stokes for incompressible lubricant. The reason behind this is the fact that based on the previously published works, for example, see $[23,27]$, significant pressure gradients in the surface-normal direction may exist due to large dimple depth as employed here, and consequently, Reynolds theory produces an incorrect prediction of the hydrodynamic pressure profile. Therefore, in this work the Reynolds-averaged Navier-Stokes (RANS) equation coupled with the continuity equation are adopted to obtain the hydrodynamic pressure profile. It should be noted that in the present study, in order to obtain more accurate results, for all following computations the turbulence model is employed. The reason behind this is that according to the work of Song et al. [28], the values of viscous and inertia forces change in particular when the slip condition in the bearing presents. To simplify the numerical simulation processing, the viscositytemperature characteristics of lubrication are ignored. 
The RANS equation is:

$$
\frac{\partial}{\partial x_{i}}\left(\rho u_{i} u_{j}\right)=-\frac{\partial p}{\partial x_{i}}+\frac{\partial}{\partial x_{j}}\left[\mu \frac{\partial u_{i}}{\partial x_{j}}-\rho \overline{u_{i}^{\prime} u_{j}^{\prime}}\right]
$$

The continuity equation is:

$$
\frac{\partial}{\partial x_{i}}\left(\rho u_{i}\right)=0
$$

In Eqs. (1-2), $\rho$ refers to the density of the fluid; $u_{\mathrm{i}}$ and $u_{\mathrm{j}}$ denotes the average velocity components for $x, y, z ; p$ expresses the hydrodynamic pressure; $\mu$ denotes the viscosity; $u_{\mathrm{i}}{ }^{\prime}$ and $u_{\mathrm{j}}^{\prime}$ express the fluctuation velocities; - $\rho \overline{u_{i}{ }^{\prime}{ }^{\prime}{ }_{j}}$ is the Reynolds stress; and $i, j=1,2,3$ $(x, y, z)$. In this work, the Reynolds stress is solved by the standard $k$ and $e$ models [29], where $k$ is turbulent kinetic energy and $e$ turbulent dissipation rate.

\subsection{Slippage modelling}

The slippage boundary on the contacting surface can be promoted by modifying the roughness and wetting properties of the surface $[9,18]$. In this way, the no-slippage condition assumption is no longer valid. The Navier-slippage model as expressed in Eq. (3) is adopted in the present work. In this model, the slippage length $\beta$ is employed to assign the relation between slippage velocity $u_{\mathrm{s}}$ and surface shear rate $\partial u /\left.\partial z\right|_{\text {surface }}$.

$$
u_{s}=\left.\beta \frac{\partial u}{\partial z}\right|_{\text {surface }}
$$

For all following simulations, to simulate the effect of a slippage in a deterministic way, a user-defined-function (UDF) is developed to in the ANSYS FLUENT ${ }^{\circledR}$ package. As a note, UDF is an additional subroutine that allows a user to define the boundary conditions and material properties as well as to specify customized model parameters. In the present analysis, in order to achieve the optimal behavior of slippage surface, the slippage length is set to 100 $\mu \mathrm{m}$ based on the work of Choo et al. [30].

\subsection{Cavitation Model}

As widely known, during the operation of the journal bearing, the cavitation may occur, especially in the divergent area. In this study, for all following computations, the cavitation phenomenon in the lubricant domain is modelled by using a multiphase cavitation model, which considers that cavitation results from the pressure change. Additionally, the cavitation model adopted here allows the growth of gas bubbles which often accompanies the cavitation process is calculated. Theoretically, the cavitation and fluid models are coupled through momentum and single set of density equations for the mixture. As a note, the validity of the multiphase cavitation model employed here has been experimentally demonstrated in previously published work [26]. In the present work, the multiphase cavitation model of Zwart-Gelber-Belamri is used due to their capability (less sensitive to mesh density, robust and converge quickly) $(29,31)$.

In cavitation, the liquid-vapor mass transfer (evaporation and condensation) is governed by the vapor transport equation [29,31]:

$$
\frac{\partial}{\partial t}\left(\alpha_{v} \rho_{v}\right)+\nabla \cdot\left(\alpha_{v} \rho_{v} \mathrm{v}\right)=R_{g}-R_{c}
$$

where $\alpha_{v}$ is vapor volume fraction and $\rho_{v}$ is vapor density. $R_{g}$ and $R_{c}$ account for the mass transfer between the liquid and vapor phases in cavitation. For Zwart-Gelber-Belamri model assuming that all the bubbles have the system size in a system, the final form of the cavitation is as follows [29]:

$$
\begin{aligned}
& p \leq p_{v}, R_{g}=F_{\text {evap }} \frac{3 \alpha_{\text {nuc }}\left(1-\alpha_{\mathrm{v}}\right) \rho_{\mathrm{v}}}{R_{\mathrm{B}}} \sqrt{\frac{2}{3} \frac{\mathrm{P}_{\mathrm{v}}-\mathrm{P}}{\rho_{\ell}}} \\
& p \geq p_{v}, R_{c}=F_{\text {cond }} \frac{3 \alpha_{\mathrm{v}} \rho_{\mathrm{v}}}{R_{\mathrm{B}}} \sqrt{\frac{2}{3} \frac{\mathrm{P}-\mathrm{P}_{\mathrm{v}}}{\rho_{\ell}}}
\end{aligned}
$$

where $F_{\text {evap }}=$ evaporation coefficient $=50, F_{\text {cond }}=$ condensation coefficient $=0.01, R_{B}=$ bubble radius $=10^{-6} \mathrm{~m}, \alpha_{\text {nuc }}=$ nucleation site volume fraction= $5 \times 10^{-4}, \rho_{l}=$ liquid density and $p_{v}=$ vapor pressure.

\subsection{CFD Model}

Recent evidence suggests that in order to improve the load support and reduce the coefficient of friction, the partial texturing must be employed. The model geometry of partially textured journal bearing studied here is inspired to the geometry employed in [4].

In order to achieve the effective computational time, two-dimensional textured bearing geometry and the corresponding groove texture are used as shown in Fig. 1. A groove cell is characterized by its depth $(d)$ and width ( $\left.W_{\text {texture }}\right)$. For all following simulations considered here, it is assumed that the $W_{\text {texture }}$ is constant and equal to $4 \mathrm{~mm}$, while 
the distance between grooves ( $\left.W_{\text {gap }}\right)$ is fixed at a value of $0.225 \mathrm{~mm}$. A series of ten "half-round" grooves applied on the certain area of the bearing surface is adopted. The reason to choose the "half-round" shape of groove is that it is easier to manufacture it in practice. Additionally, based on literature review there is a little effect of the groove shape on the tribological performances of the textured surface. In this work, grooves are introduced from angular coordinates of $\theta_{i}=122^{\circ}$ measured from the position of maximum film thickness. Based on the previous study [4] on textured journal bearing, locating grooves at the maximum pressure zone, which is similar in this study, results in a significant reduction in the friction coefficient.

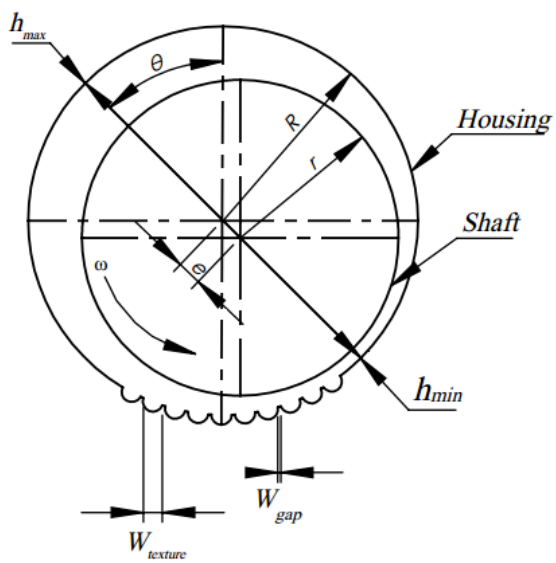

(a)

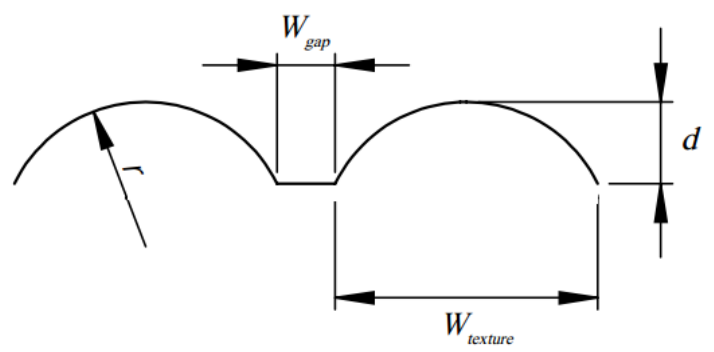

(b)

Fig. 1. (a) Textured journal bearing geometry, (b) two-dimensional groove texture.
Therefore, in order to complement their findings, more attention has been paid to optimizing texturing parameters with respect to the enhancement of the load support. In the present work, the variation of the groove deep is conducted at different loading conditions (i.e. low, medium, and high loading) and surface characteristics (i.e. with and without slippage promotion).

For all following computations, a textured bearing model by using a cosine profile is employed as shown in Fig. 2. The profile of the lubricant film thickness $h$ of the journal bearing used here reads: $h=\mathrm{c}(1+\varepsilon \cos \theta)[32,33]$. The bearing model depicted in Figs. 1-2 has the following dimensions: the shaft radius $r$ is 50 $\mathrm{mm}$, the housing radius $R$ is $50.145 \mathrm{~mm}$, and the angular velocity of shaft is $48.1 \mathrm{rad} / \mathrm{s}$. For lubricant properties, the dynamic viscosity $\mu$ used is 0.0127 Pas, and the density of lubricant $\rho$ is $840 \mathrm{~kg} / \mathrm{m}^{3}$, while for the lubricant vapor the vaporization pressure used is $20 \mathrm{kPa}$, and the dynamic viscosity is $2 \times 10^{-5} \mathrm{Pas}$ and its density to $1.2 \mathrm{~kg} / \mathrm{m}^{3}$.

In the present simulation, as shown in Fig. 2 the boundary condition of the model is set as follows: One side of the maximum film thickness is used as an inlet and the other as an outlet. The pressure at the inlet $\left(P_{\text {in }}\right)$ and outlet $\left(P_{\text {out }}\right)$ boundaries is taken as the ambient pressure, i.e. zero pressure. The upper surface of the lubricant film is modelled as a stationary wall, while the lower surface is modelled as a moving wall with no-slip condition and a rotational velocity equal to that of the shaft. During the simulation in ANSYS FLUENT® software, the location of cavitation and the pressure profile are calculated in the MIXTURE model.

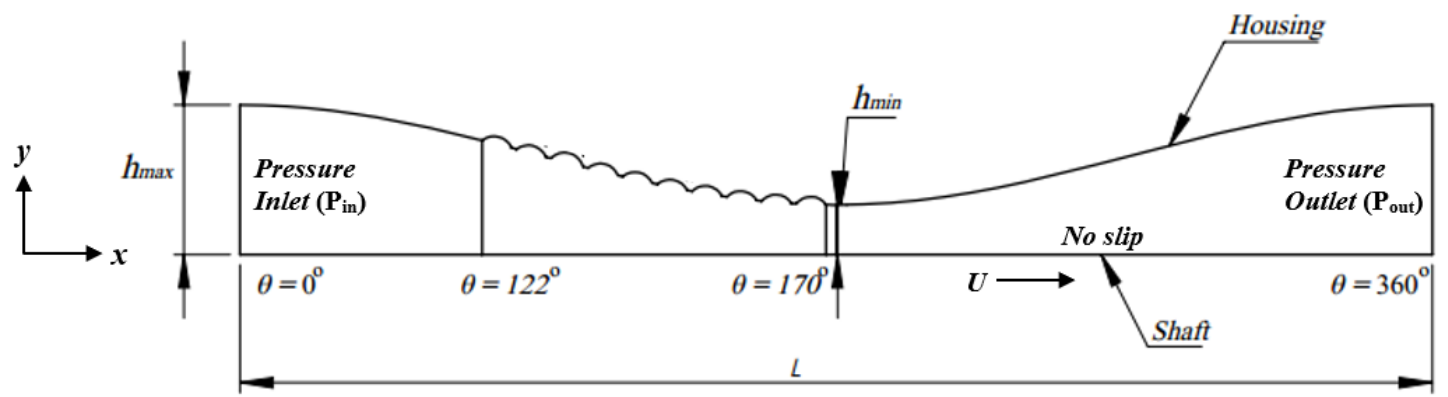

Fig. 2. Textured bearing modelled as a cosine profile. 


\subsection{Solution Setup}

Before the solution of the bearing performance, the meshing of lubrication film needs to be performed. The computational domain is meshed with the uniform quadrilateral grid. In this way, the grid system can minimize the relative percentage difference of the simulation result up to no more than $1 \%$ at the convergence criteria for the velocity parameter (set to be $1.0 \times 10^{-6}$ ), which is adequate for engineering calculation in particular of bearing. Additionally, more refinement of the grid system in particular in the dimple area is also performed in order to get mesh independent, see Fig. 3, for example. From the figure, it can be observed that if the mesh number is above 80,000 , the simulation results do not differ anymore. But obviously the computational cost increases. Therefore, the mesh number used for all following situations is approximately 80,000 - 90,000 nodes across the film depending on the groove depth.

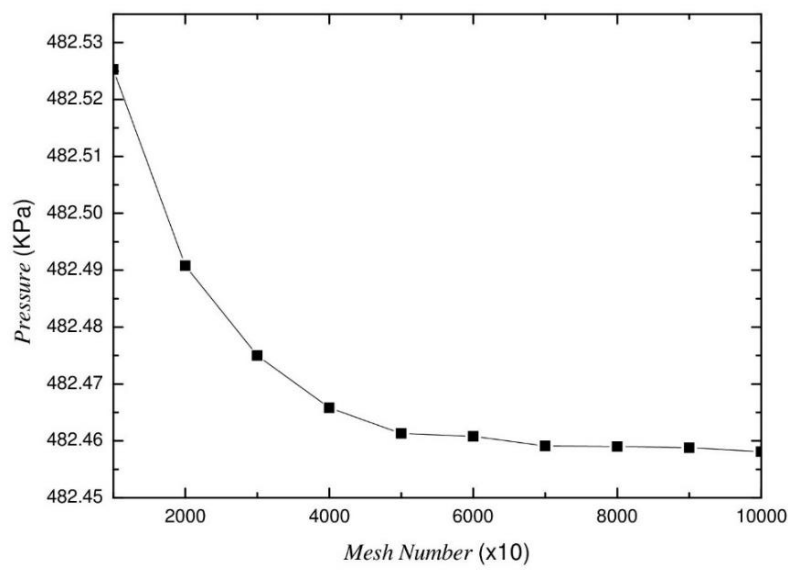

Fig. 3. Independent mesh of the model.

In the present numerical analysis, the pressurebased solver is adopted. The pressure-velocity coupling is treated using the SIMPLEC algorithm, while for the momentum equations the second order upwind discretization scheme is chosen. For the volume fraction equation, the turbulent kinetic energy as well as the turbulent dissipation rate, the QUICK discretization scheme is chosen. For more accurate result, a convergence tolerance of $1 \times 10^{-6}$ is employed for all residual terms.

In the present study, the hydrodynamic pressure as well as the load support is of particular interest. Once the hydrodynamic pressure is solved through Eq. (1), the load support of the bearing can be calculated from the integration of the hydrodynamic pressure acting on the journal as indicated in Fig. 2. It reads:

$$
F=\int_{0}^{L} p(x) d x
$$

where $F$ refers to load support.

\section{RESULTS AND DISCUSSION}

In the present work, the simulation has been carried out for two main configurations of partially textured journal bearing as described in Table 1 . For the first configuration, that is, purely textured bearing, the prediction of the tribological performance is calculated when the no-slippage situation is assumed. Emphasis has been given to the second configuration which are calculated for textured surface combined with artificial slippage. This is of particular interest because it is believed that based on several recent studies, including [14-17], the textured surface modification with proper slippage pattern may lead to a significant improvement of their load support, achieving in certain cases an increase of $200 \%$ in comparison to conventional designs. However, in their works, the single-phase cavitation model was still used.

For two configurations of bearing studied here, the effect of the groove depth will be discussed further in this work. As widely known, the groove depth is a critical design parameter, important for the performance of textured bearing. In this work, two categories of the groove are introduced in terms of minimum film thickness $h_{\min }$, i.e. deep groove (i.e. $d / h_{\min }=4.5$ and 6 in this case) and shallow grooves $\left(d / h_{\min }=\right.$ 0.45 and 1). It should be pointed out that the variation of the groove depth is carried out such that the groove depth does not exceed half of the groove width.

Table 1. Simulated type of contact.

\begin{tabular}{|l|c|c|}
\hline Configuration & $\begin{array}{c}\text { Type of } \\
\text { surface }\end{array}$ & $\begin{array}{c}\text { Ratio of groove } \\
\text { depth to minimum } \\
\text { film thickness } \\
\left(\boldsymbol{d} / \boldsymbol{h}_{\text {min }}\right)\end{array}$ \\
\hline Configuration 1 & Pure texturing & $0.45 ; 1 ; 4.5 ; 6$ \\
\hline Configuration 2 & $\begin{array}{c}\text { Combined } \\
\text { texture and } \\
\text { slippage }\end{array}$ & $0.45 ; 1 ; 4.5 ; 6$ \\
\hline
\end{tabular}




\subsection{Validation}

Before the investigation of the texturing effect (with/without slippage) on the journal bearing performance is explored, it is necessary to check the validation of the CFD solution setup of bearing model as well as the multiphase cavitation model implemented in this simulation. A comparative study in terms of the hydrodynamic pressure of the smooth bearing between the simulation data in literature [4] and the present work is performed for the validation purpose. The geometry as well as the operating condition simulated in the present work is similar to those in literature [4].

Based on Fig. 4, the hydrodynamic pressure predicted by the present study matches well with the corresponding simulation results in the literature [4], which indicates the validation of the used solution setup (i.e. bearing model and cavitation model). Therefore, all following simulations are carried out based on the cosine profile model.

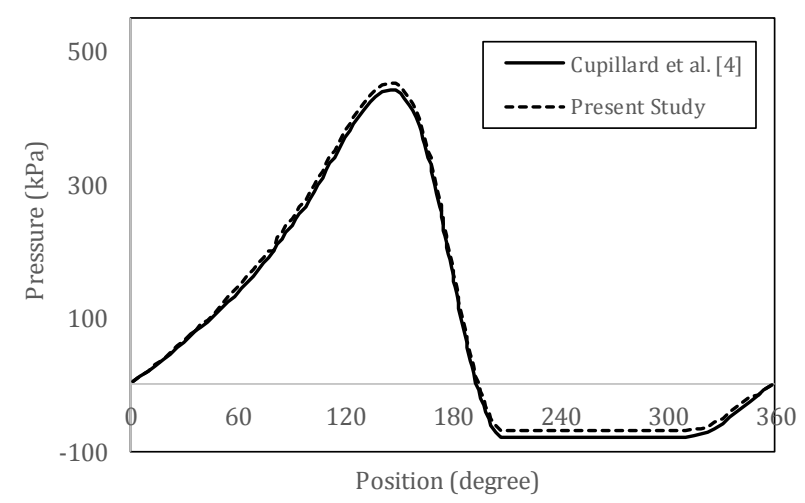

Fig. 4. The comparison of hydrodynamic pressure between the simulation by present study and that by Cupillard et al. [4].

\subsection{Pure Texturing}

As widely known, the lubrication bearing performance is directly influenced by the eccentricity ratio representing the loading during operation. Therefore, in the present study the prediction of the performance is also made for different eccentricity ratios, i.e. $\varepsilon=0.2$ (light loading), $\varepsilon=0.5$ (medium loading) and $\varepsilon=0.8$ (heavy loading).

Figure 5 shows the hydrodynamic pressure of the textured and smooth journal bearings for light loading (i.e. low eccentricity ratio). It can be seen there is a shift of the location of the maximum pressure if the texturing is used. The results also show that the textured journal bearing operates with lower value of maximum hydrodynamic fluid film pressure in comparison to smooth journal bearing. However, when the textured surface is introduced, the length of the cavitation area becomes shorter. As a consequence, the improvement of the performance of the textured bearing is highlighted. The results of the load support of the textured bearing become higher compared to that of smooth case. Depending the ratio of depth to minimum film thickness $d / h_{\min }$, the increase in load support ranges from $34 \%$ to $50 \%$. With respect to the effect of groove depth, it can be found that the pressure profiles have a similar trend in terms of cavitation zone. Clearly, based on physical point of view the promotion of surface texturing is able to reduce the cavitation phenomena irrespective of the groove depth.

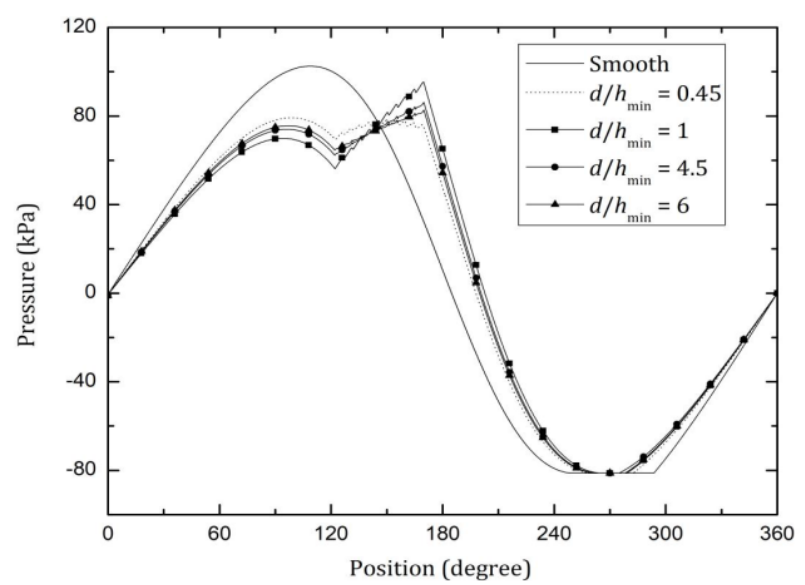

Fig. 5. Hydrodynamic pressure distributions for low loaded bearing $(\varepsilon=0.2)$.

Further, from Fig. 5, one can find that the textured bearing, compared to the smooth bearing, induces the valley located at the starting position of the groove. This prevails for all value of groove depths. In the case of textured bearings, there exists jump value in the maximum hydrodynamic pressure at the circumferential angle of about $170^{\circ}$. This result may be explained by the fact that there is a sudden change in the film thickness at the location of the end position of the groove. The most interesting finding is that there is an optimum value for $d / h_{\text {min }}$ for texturing case. It is evident from Fig. 5 that the $d / h_{\min }$ of 1 generates the highest load support which indicates that for low loaded bearing the use of shallow dimple is recommended for improving the load support. 
Figures 6 and 7 provide a comparison of the hydrodynamic pressures between the smooth bearing and the textured one for medium (i.e. $\varepsilon=0.5)$ and heavy loadings $(\varepsilon=0.8)$, respectively. Comparing Figs. 6 and 7 shows that the cavitation zone of the high loaded bearing covers $30 \%$ larger than that of the medium loaded bearing. For the case of medium loaded bearing, the cavitation occurs at the circumferential angle of about $230^{\circ}$. When the eccentricity ratio is increased to be 0.8 , there is a shift of the starting location of the cavitation toward the inlet of the contact. Generally, to compensate the decrease in the pressure due to the cavitation, the lubricant in the convergent area must produce more hydrodynamic pressure as reflected in Figs. 6 and 7. As expected, the peak pressure increases with the increase in the eccentricity ratio for all cases (i.e. smooth and textured contact).

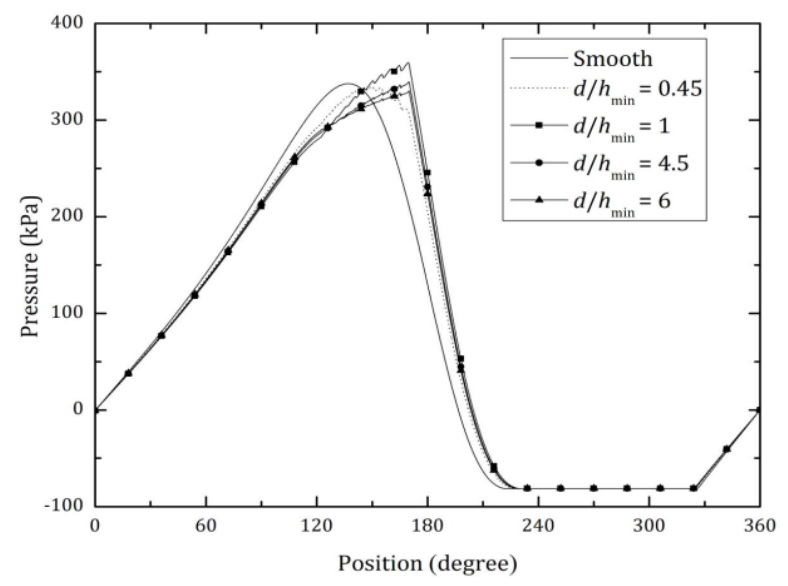

Fig. 6. Hydrodynamic pressure distributions for medium loaded bearing $(\varepsilon=0.5)$.

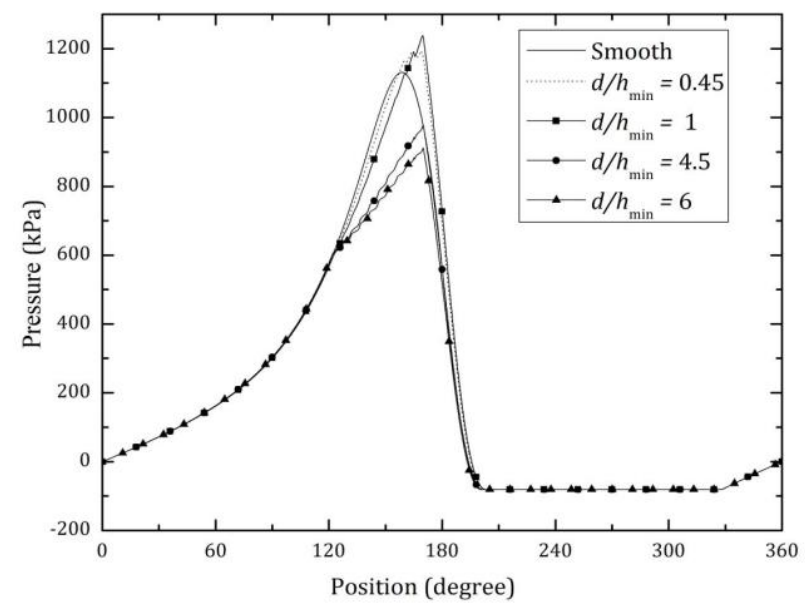

Fig. 7. Hydrodynamic pressure distributions for heavy loaded bearing $(\varepsilon=0.8)$.
It is interesting to note that comparing the three results in Fig. 5 (i.e. low loaded bearing), Fig. 6 (i.e. medium load bearing), and Fig. 7 (i.e. heavy loaded bearing) the textured bearings generate the larger pressure profile compared to the smooth one. Surprisingly, for textured bearing with $d / h_{\min }$ of 1 the peak pressure reaches the highest value compared to other patterns irrespective of the eccentricity ratio. It indicates that the groove depth which is set equal to the minimum film thickness is the optimal value of groove depth for inducing the highest load support. Further analysis shows that unlike the low loaded bearing, the medium load bearing as well as the high loaded bearing with texturing flatten the hydrodynamic pressure locally and maximum pressure is influenced. In the area of maximum pressure, the pressure profile is truncated without presenting the valley of pressure.

By comparing the maximum value of fluid film pressures for three cases studied here, it has been found that the higher the loading of the bearing operation, the higher the predicted maximum hydrodynamic pressure both for the case of smooth journal bearing and textured one. The most possible explanation is that the journal bearing with high eccentricity ratio $\varepsilon$ generates more converging and diverging fraction for hydrodynamic action compared to that with low $\varepsilon$. Generally speaking, from these pressure profiles depicted above, it has been easily investigated the effect of textured surfaces as well as the groove depth on the fluid film hydrodynamic pressure distribution along the circumferential direction of the corresponding bearings. For each eccentricity ratio, it can be observed that the truncation profile is dependent on the groove depth. For deep groove, the truncation in the pressure profile seems flatter compared to the cases with shallow groove. For example, in the case of $\varepsilon=$ 0.5 when the ratio of the groove depth to the minimum film thickness $d / h_{\min }$ is increased by a factor of $10\left(d / h_{\min }=4.5\right.$ in this case $)$, the truncation in the pressure distribution becomes flatter. However, for optimal groove depth, that is, $d / h_{\min }=1$, a unique feature is observed, that is, the peak pressure is found to be highest compared to other depths. This is the reason why the largest load support predicted by the bearing with $d / h_{\min }$ of 1 can be achieved. 
In more detail, the effect of groove depth at different eccentricity ratios on the load support is shown in Fig. 8. Here, the result is presented in terms of performance ratio, that is, the ratio of the load support predicted by the textured bearing to that smooth bearing. The negative value means that the deterioration of the bearing performance is noted. Figure 8 illustrates that the texturing effect becomes more dominant for the case of low eccentricity ratio (i.e. low load) irrespective of the groove depth. A contradictive result is observed for heavy loaded bearing. The use of texturing does not affect the load support very much for each groove depth. Based on the physical point of view, the large eccentricity ratio can weaken the hydrodynamic action of the texturing because of enhancement in the cavitation phenomena in the divergent area. In fact, for deep groove $\left(d / h_{\min }>1\right.$ in this study) the negative effect of texturing on the load support can be clearly highlighted for high $\varepsilon$. The reduce in load support, compared to the smooth bearing, reaches $14 \%$ for deep groove. It indicates that for the case of high loaded bearing (i.e. high eccentricity ratio) the use of textured surface with deep groove is not so effective in improving load support.

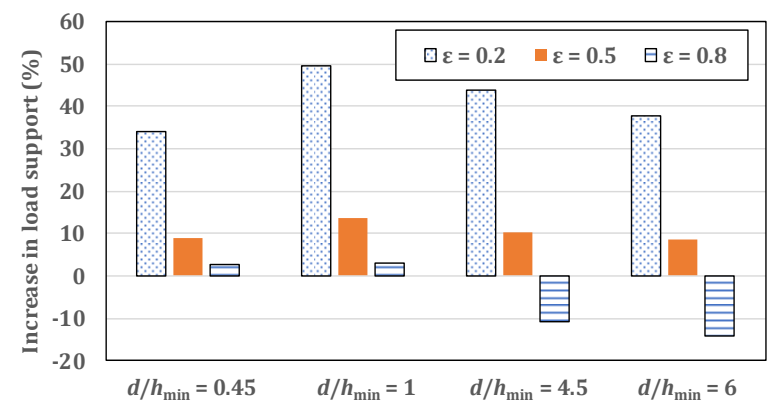

Fig. 8. Effect of texturing on the load support performance varying the eccentricity ratios for different $d / h_{\min }$. (Note: The performance prediction is evaluated in comparison with the smooth conventional bearing).

By further inspecting Fig. 8, it can be seen that an optimum groove depth with respect to the load support is identified for all cases studies here. On the other words, an interesting way of improving the load support of partially textured bearing is by designing the groove depth equal to the minimum film thickness, that is, $d=h_{\min }$ as mentioned earlier. Based on physical point of view, this behavior is more due to the effect of the presence of the truncation in the maximum pressure area rather than the cavitation phenomena as demonstrated in Figs. 5-7.

\subsection{Slip Texturing}

As discussed by Zhang et al. [34], the arrangement of slippage boundary on smooth surface of journal bearing has a crucial effect on the tribological performance including the occurence of cavitation. However, the available literature survey indicates that the studies related to slippage arrangement on textured surface of journal bearing are very limited. Therefore, it is also interesting to check whether a different placement of the artificial slippage applied on textured contact has a significant effect on the tribological performance. In the present study in order to further explore the advantage of the existence of the fluid-solid interface slippage, in the following computations the predicted performance simultaneously will be evaluated.

In the present work, three placements of artifical slippage, applied to create three hydrophobic textured patterns (Fig. 9), are proposed and compared with each other. With respect to the load support enhancement, the textured surface with shallow groove $\left(d / h_{\min } \leq 1\right)$ is chosen based on the results discussed in the previous section.

Figure 10 shows the influence of slippage placements applied of textured bearing on the hydrodynamic pressure for two different values of the groove depth: $d / h_{\min }=0.45$ and $d / h_{\min }=1$. In this analysis, the performance of slippage textured bearing is compared to the solely texture bearing (without slippage) to assess the influence of slippage promotion. As illustrated in Fig. 10 (a), in the case of $d / h_{\min }=0.45$, the slippage-textured bearing with "slip gap" pattern is characterized by substantially same level of peak pressure as well as the length of the cavitation zone, in comparison to the solely textured bearing. It is also found that a very large increase in the hydrodynamic pressure profile is registered when the slippage is promoted at more edges of the groove cells, that is, "slip texture" and "slip full". For such patterns, the pressure increases and then decreases to minimum value along the convergent zone. However, at textured area the pressure makes a pressure jump which yields the peak pressures for "slip texture" and "slip full" patterns become higher compared to "slip gap" and "no slip" patterns. Additionally, the most interesting finding based on Fig. 10 (a) is that the length of the cavitation section reduces a lot at the divergent zone. 


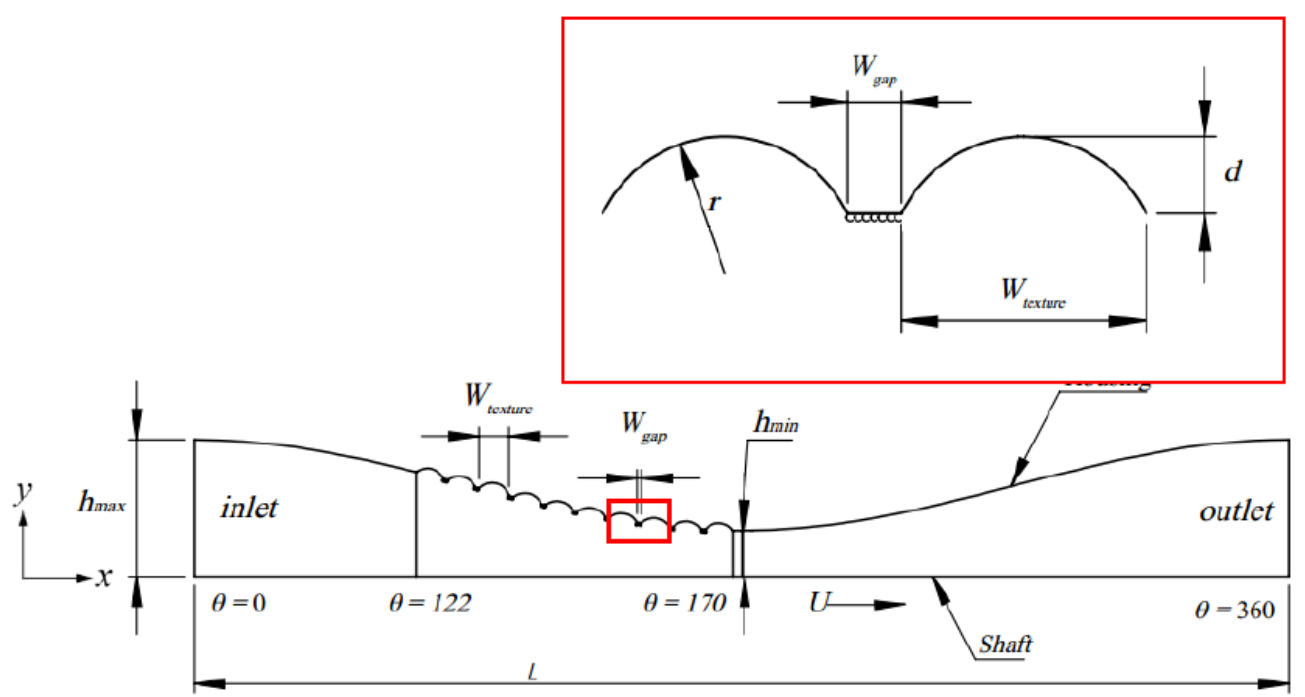

(a)

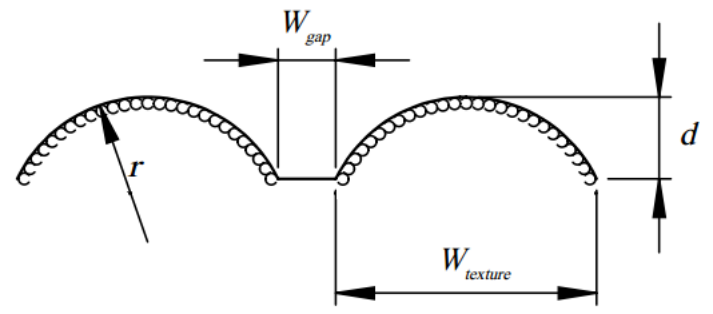

(b)

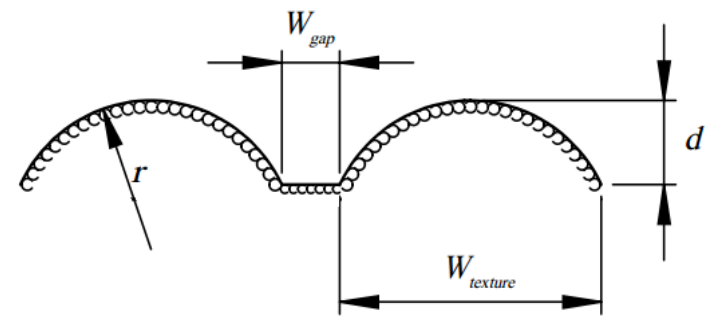

(c)

Fig. 9. Three placements of slippage on textured surface, (a) "Slip Gap" (i.e. slippage covers only valley edge of the groove), (b) "Slip Texture" (i.e. slippage applied on the internal edge of the groove), (c) "Slip Full" (i.e. slippage applied on all edges of the groove).

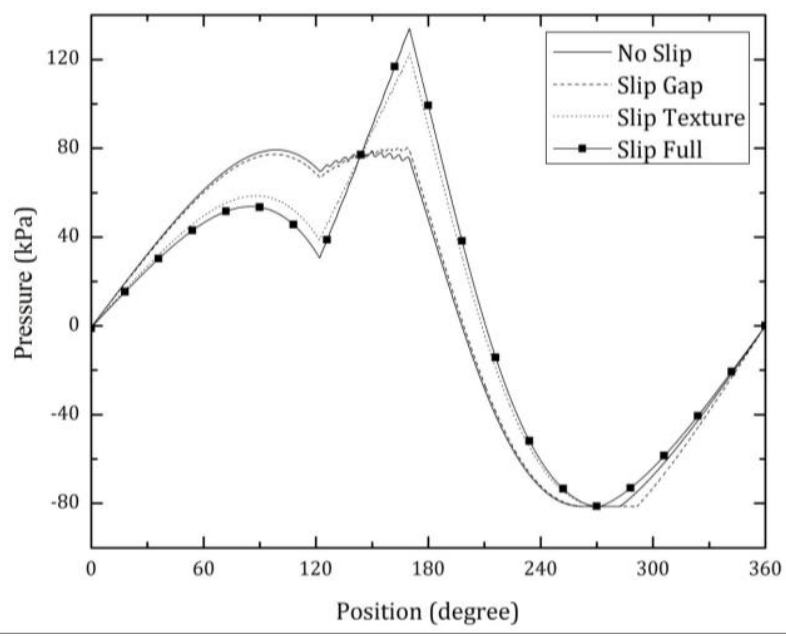

(a)

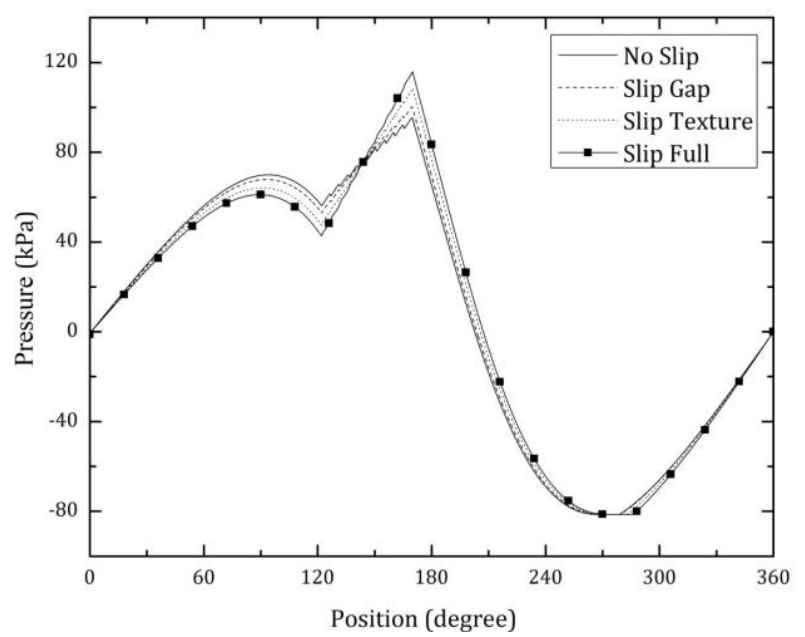

(b)

Fig. 10. Hydrodynamic pressure distributions of textured bearing at different slippage arrangements for the case: (a) $d / h_{\min }=0.45$, (b) $d / h_{\min }=1$. All results are evaluated at $\varepsilon=0.2$. 
Based on physical point of view, it indicates that more (super)oleophobic materials used to mimic the slippage on textured area generate more positive effects in enhancing the pressure profile. This finding matches well with the results of Muchammad et al. [18] who analytically concluded that employing more slippage could retard cavitation level. As indicated in Fig. 10, the pressure rise is due to a shorter cavitation region at the divergent area, while at the convergent area in particular at the textured area, the "slip full" textured region generates higher pressure gradient.

In the case of $d / h_{\min }=1$, based on Fig. 10b two specific features can be drawn. First, in general in comparison with the purely textured bearing, the slippage-textured bearings give the similar performance both at the convergent zone and the divergent zone. The difference lies only in the value of peak pressure depending on the groove depth. Second, it is very interesting to note that compared to the case of $d / h_{\min }=0.45$ (Fig. 10a), the peak pressure for "slip texture" as well as "slip full" case becomes lower. It indicates that introducing more slippage condition on textured zone does not affect the performance very much. This is different when the groove with $d / h_{\min }$ of 0.45 is employed; the more (super)oleophobic material used on textured area, the larger the corresponding load support becomes. It should be noted that based on Fig. 10, the predicted load support of slippagetextured bearing will be a function of pressure in textured area and pressure distribution in the cavitation zone. For more details, the load support predicted by textured bearing with or without slippage for two different groove depths is summarized in Fig. 11.

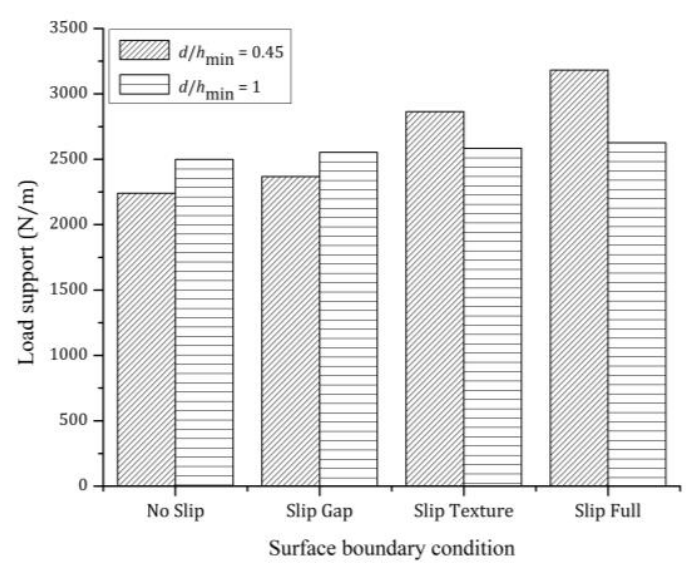

Fig. 11. The load support of textured bearing at different surface conditions for two values of groove depth. All results are evaluated at $\varepsilon=0.2$.
Obviously, the results suggest that: (1) if the textured bearing is designed to have a very shallow deep (for example, $d / h_{\min }=0.45$ as studied here), for achieving the maximum load support it is very advisable to use more slippage conditions on groove cells (i.e. full slip), (2) if the textured bearing with higher groove depth is employed $\left(d / h_{\min }=1\right.$, for instance), the use of the slippage is not necessary; the texturing itself is able to provide the load support.

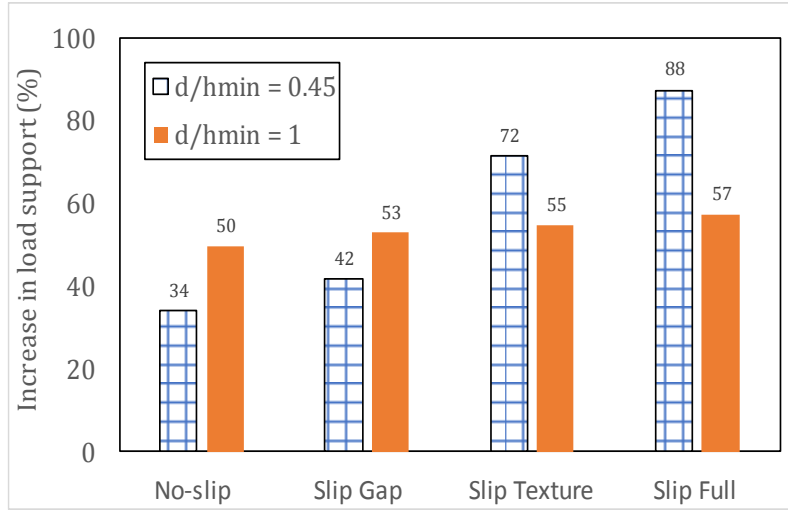

Fig. 12. Effect of slippage placement on the load support performance of the low loaded bearing. Note: The performance prediction is evaluated in comparison with the conventional smooth bearing.

Finally, it is necessary to investigate the load support improvement in comparison with the conventional bearing (without texturing and/or slippage) for various possible textured surfaces. Figure 12 shows the effect of texturing application with various slip conditions for two values of shallow groove depths, i.e. $d / h_{\min }=0.45$ and $d / h_{\min }=1$ on the load support. All calculations are compared with the performance of the conventional bearing (i.e. smooth without slippage). It can be seen that when the improvement in load support is superior for the purely textured bearing (50 \% larger compared to smooth bearing), the slippage-textured bearing with the same groove depth (in this case $d / h_{\min }=1$ ) produces slightly more load support (only up to $7 \%$ larger) compared to purely textured bearing. As mentioned earlier, it indicates that the texturing with $d / h_{\min }=1$ has a more dominant effect than slippage effect. A different result is shown for lower groove depth (i.e. $d / h_{\min }=0.45$ ). The textured bearing with lower groove depth yields a very significant increase in the load support by adding the slippage boundary condition on groove cells. For example, for case of "slip full", the $88 \%$ and 40 $\%$ improvements of load support are obtained 
compared to conventional bearing and purely textured bearing, respectively. In fact, as illustrated in Fig. 8, for low loaded bearing the purely textured bearing with $d / h_{\min }=0.45$ gives lowest increase in load support (only up to 34 $\%$ ) compared to other textured configurations. Generally speaking, these results demonstrate how slippage combined with texturing can be very promising way for designing very high load support lubricated bearing in certain conditions.

To validate the above conclusion about the advantages of the combining texturing with slippage in the case of low loaded bearing, the tribological analysis of medium and heavy loaded textured bearings is also conducted. For this, the eccentricity ratio is changed to be 0.5 and 0.8 , respectively, while other input parameters for these calculations are the same as ones employed for low loaded bearing analysis.

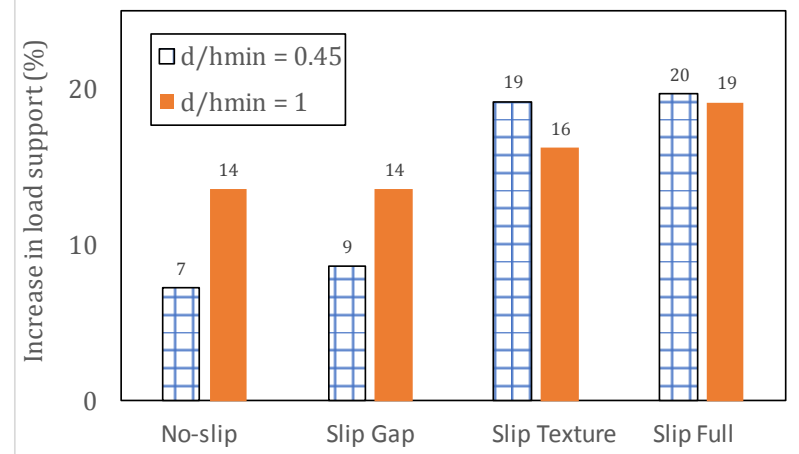

Fig. 13. Effect of slippage placement on the load support performance of medium loaded bearing $(\varepsilon=$ $0.5)$. Note: The prediction is evaluated in comparison with the smooth conventional bearing.

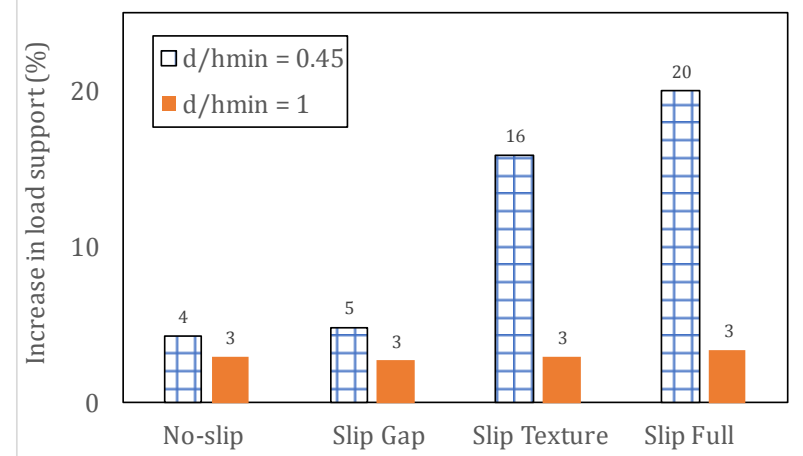

Fig. 14. Effect of slippage placement on the load support performance of heavy loaded bearing $(\varepsilon=$ $0.8)$. Note: The prediction is evaluated in comparison with the conventional smooth bearing.

Figure 13 and 14 provide the results of load support for slippage-textured bearing under medium loading (i.e. $\varepsilon=0.5$ ) and heavy loading (i.e. $\varepsilon=0.5$ ), respectively. Two specific features can be observed based on these figures. First, in general with respect to the performance of conventional smooth bearing the increase in load support of the medium loaded bearing as well as the heavy loaded one is not as large as that of the low loaded bearing. It seems that the positive effect of texturing reduces with the increase in the eccentricity ratio. Obviously, this behavior is similar to the performance of purely textured bearing. Second, with respect to the effect of groove depth, the bearing with very shallow groove (i.e. $d / h_{\min }=0.45$ ) results in more significant improvement in the load support compared to that with deeper shallow $\left(d / h_{\min }=1\right.$ in this case $)$ when more slippage conditions are induced on textured area. For example, for the case of "slip full" the difference in performance is quite large. For pattern with $d / h_{\min }=1$, the increase in load support achieves $20 \%$ while for $d / h_{\min }=1$, the increase is just up to $3 \%$. The latter gives the same performance with the purely textured one. On the other words, as discussed earlier, combining the slippage with deeper groove depth is less effective to produce the load support for all loading conditions considered here.

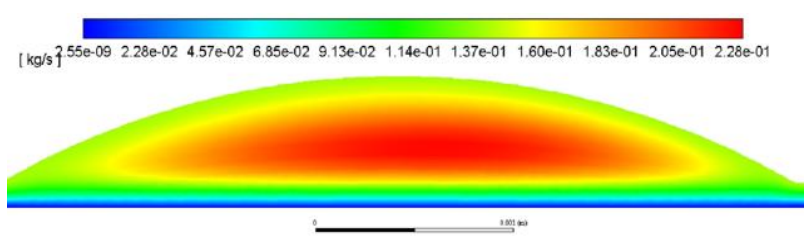

(a)

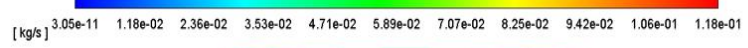

(b)

Fig. 15. Stream function in the dimple geometry of purely textured bearing with $d / h_{\min }=4.5$ for case: (a) $\varepsilon=0.2$, (b) $\varepsilon=0.8$.

To investigate why texturing with/without slip is more effective at low loaded bearing in terms of load support, one needs to understand the main factors contributing the hydrodynamic pressure. Two specific factors can be drawn. First, in the case of low loaded bearing (i.e. low eccentricity ratio), as indicated in Fig. 5 and Fig. 10, respectively, for pure texturing and slip-texturing cases, some additional pressure can be generated due to the dimples. The dimples seem to be able to produce a higher gradient pressure compared 
to the dimples in the case of higher eccentricity ratios (i.e. $\varepsilon=0.5$ and 0.8 as shown in Figs. 6 and 7, respectively). Second, as reflected in Fig. 15 a recirculation zone in the flow inside the dimples in the case of $\varepsilon=0.2$ is found to be larger compared to ones in the case of $\varepsilon=0.8$. It can be seen that the vortex decreases in size as the eccentricity ratio increases. This is as expected because for the same $d / h_{\min }$ the dimple depth changes following the eccentricity ratio. This difference leads to the change of flow characteristic inside the dimple. Based on the work of Shankar and Deshpande [35], a stretched recirculation zone as depicted in Fig. 15 (b) leads to lower pressure gradient and thus lower pressure generation as shown in Figs. 6 and 7.

\section{CONCLUSION}

This study investigates the hydrodynamic performance of textured journal bearings considering the slippage. Large numbers of CFD analyses are conducted to find the influence of groove depth, slippage placement, and eccentricity ratio on ultimate load support and hydrodynamic pressure. The multiphase cavitation model used here revises the traditional single-phase cavitation model that neglects the phase change within the divergent zone. Based on the simulation results, the following conclusions can be drawn from the study:

1. The simulation results show that the higher the eccentricity ratio, the lower the positive effect of introduction of texturing. This prevails both for the purely textured bearing and the slippage textured one.

2. In the case of purely textured bearing, the optimum groove depth exists to a achieve the maximum load support. For a purely textured journal bearing with given eccentricity ratio, the groove depth which is equal to minimum film thickness results in the highest additional load support.

3. In the case of a combination of slippage and texturing, a well-chosen placement of the artificial slippage condition on textured zone of the bearing has more positive effect with respect to the load support, compared to purely textured surface. Introducing the slippage boundary on all groove edges of textured area leads to a significant enhancement in load support.
4. The positive effect of the slippage-textured bearing would have been more significant in enhancing the tribological performance if a relatively shallow groove depth has been used. This finding may have useful implications for enhancing the additional load support.

For future, in order to investigate the potential application of slippage in more detail, the work will be dedicated to the comparison analysis between the texturing effect and slippage effect on the load support and friction generation.

\section{Acknowledgement}

This research is fully supported by RPI-BT (Research Publication International-High Reputation) Grant, No. 38705/UN7.P4.3/PP/2018. The authors fully acknowledged Institute for Research and Community Services (LPPM) Diponegoro University for the approved fund which makes this important research viable and effective.

\section{REFERENCES}

[1] X. Lu, M.M. Khonsari, An Experimental Investigation of Dimple Effect on the Stribeck Curve of Journal Bearings, Tribology Letters, vol. 27 , pp. $169-176,2007$, doi: $10.1007 /$ s11249007-9217-x

[2] L. Galda, J. Sep, A. Olszewski, T. Zochowski, Experimental Investigation into Surface Texture Effect on Journal Bearings Performance, Tribology International, vol. 136, pp. 372-384, 2019, doi: 10.1016/j.triboint.2019.03.073

[3] D.A. Bompos, P.G. Nikolakopoulus, Static Performance of Surface Textured Magnetorheological Fluid Journal Bearings, Tribology in Industry, vol. 37, no. 3, pp. 340345, 2015.

[4] S. Cupillard, S. Glavatskih, M.J. Cervantes, Computational Fluid Dynamics Analysis of $A$ Journal Bearing with Surface Texturing, Proceedings of the Institution of Mechanical Engineers, Part J: Journal of Engineering, vol. 222, iss. 2, pp. 97-108, 2008, doi: 10.1243/13506501JET319

[5] F.M. Meng, L. Zhang, Y. Liu, T.T. Li, Effect of Compound Dimple on Tribological Performances of Journal Bearing, Tribology International, vol. 
91, pp. 99-110, 2015, doi: 10.1016/j.triboint.2015.06.030

[6] Q. Lin, Q. Bao, K. Li, M.M. Khonsari, H. Zhao, An Investigation into the Transient Behavior of Journal Bearing with Surface Texture Based on Fluid-Structure Interaction Approach, Tribology International, vol. 118 , pp. 246-255, 2018, doi: 10.1016/j.triboint.2017.09.026

[7] D. Gropper, L. Wang, T.J. Harvey, Hydrodynamic Lubrication of Textured Surfaces: A Review of Modeling Techniques and Key Findings, Tribology International, vol. 94, pp. 509-529, 2016, doi: 10.1016/j.triboint.2015.10.009

[8] P.A. Thompson, S.M. Troian, A General Boundary Condition for Liquid Flow at Solid Surfaces, Nature, vol. 389, pp. 360-362, 1997, doi: $10.1038 / 38686$

[9] H.A. Spikes, The Half-Wetted Bearing: Part 1: Extended Reynolds Equation, Proceedings of Institution of Mechanical Engineers J-Journal of Engineering Tribology, vol. 217, iss. 1, pp. 1-14, 2003, doi: $10.1243 / 135065003321164758$

[10] A.E. Fortier, R.F. Salant, Numerical Analysis of a Journal Bearing with a Heterogeneous Slip/No-Slip Surface, ASME Journal of Tribology, vol. 127, iss. 4, pp. 820-825, 2005, doi: 10.1115/1.2033897

[11] G.J. Ma, C.W Wu, P. Zhou, Wall Slip and Hydrodynamics of Two-Dimensional Journal bearing, Tribology International, vol. 40, iss. 7, pp. 1056-1066, 2007, doi: 10.1016/j.triboint.2006.10.003

[12] L.L. Wang, C.H Lu, M. Wang, W.X Fu, The Numerical Analysis of the Radial Sleeve Bearing with Combined Surface Slip, Tribology International, vol. 47, pp. 100-104, 2012, doi: 10.1016/j.triboint.2011.10.014

[13] T.V.V.L.N. Rao, A.M.A. Rani, M. Awang, F.M. Hashim, Stability Evaluation of Three-Layered Journal Bearing with Slip/Partial Slip, Industrial Lubrication and Tribology, vol. 69, no. 3, pp. 334-341, 2017, doi: 10.1108/ILT-08-2016-0184

[14] T.V.V.L.N. Rao, A.M.A. Rani, T. Nagarajan, F.M. Hashim, Analysis of Slider and Journal Bearing using Partially Textured Slip Surface, Tribology International, vol. 56, pp. 121-128, 2012, doi: 10.1016/j.triboint.2012.06.010

[15] F. Aurelian, M. Patrick, H. Mohamed, Wall Slip Effects in (Elasto) Hydrodynamic Journal Bearings, Tribology International, vol. 44, iss. 78, pp. 868-877, 2011, doi: 10.1016/j.triboint.2011.03.003

[16] M. Tauviqirrahman, R. Ismail, J. Jamari, D.J. Schipper, Combined Effect of Texturing and Boundary Slippage in Lubricated Sliding
Contacts, Tribology International, vol. 66, pp. 274-281, 2013, doi: 10.1016/j.triboint.2013.05.014

[17] M. Tauviqirrahman, R. Ismail, J. Jamari, D.J. Schipper, A Study of Surface Texturing and Boundary Slip on Improving the Load Support of Lubricated Parallel Sliding Contacts, Acta Mechanica, vol. 224, iss. 2, pp. 365-381, 2013, doi: 10.1007/s00707-012-0752-7

[18] M. Muchammad, M. Tauviqirrahman, J. Jamari, D.J. Schipper, An Analytical Approach on the Tribological Behaviour of Pocketed Slider Bearings with Boundary Slip Including Cavitation, Lubrication Science, vol. 29, iss. 3, pp. 133-152, 2017, doi: 10.1002/ls.1361

[19] S. Ismail, M. Sarangi, Effects of Texture Shape and Fluid-Solid Interfacial Slip on The Hydrodynamic Lubrication Performance of Parallel Sliding Contacts, Proceedings of the Institution of Mechanical Engineers, Part J: Journal of Engineering, vol. 228, iss. 4, pp. 382-396, 2014, doi: $10.1177 / 1350650113511960$

[20] Q. Lin, Z. Wei, N. Wang, W. Chen, Effect of LargeArea Texture/Slip Surface on Journal Bearing Considering Cavitation, Industrial Lubrication and Tribology, vol. 67, no. 3, pp. 216-226, 2015, doi: 10.1108/ILT-05-2013-0055

[21] L. Biancofiore, M. Giacopini, D. Dini, Interplay Between Wall Slip and Cavitation: A Complementary Variable Approach, Tribology International, vol. 137, pp. 324-339, 2019, doi: 10.1016/j.triboint.2019.04.040

[22] M.K. Ghosh, B.C. Majumdar, M. Sarangi, Theory of Lubrication. McGraw Hill Education, Bengaluru, 2012.

[23] M.B. Dobrica, M. Fillon, About the Validity of Reynolds Equation and Inertia Effects in Textured Sliders of Infinite Width, Proceedings of the Institution of Mechanical Engineers, Part J: Journal of Engineering Tribology, vol. 223, no. 1, pp. 69-78, 2009, doi: 10.1243/13506501JET433

[24] Y. Mao, L. Zeng, Y. Lu, Modeling and Optimization of Cavitation on a Textured Cylinder Surface Coupled with the Wedge Effect, Tribology International, vol. 104 , pp. $212-224,2016$, doi: 10.1016/j.triboint.2016.09.002

[25] F. Concli, Pressure distribution in Small Hydrodynamic Journal Bearings Considering Cavitation: A Numerical Approach Based on the Opensource CFD Code OPENFOAM®, Lubrication Science, vol. 28, iss. 6, pp. 329-347, 2016, doi: $10.1002 /$ ls. 1334

[26] D.Y. Dhande, D.W. Pande, Multiphase Flow Analysis of Hydrodynamic Journal Bearing using CFD 
Coupled Fluid Structure Interaction Considering Cavitation, Journal of King Saud University Engineering Sciences, vol. 30, iss. 4, pp. 345-354, 2018, doi: 10.1016/j.jksues.2016.09.001

[27] J. Li, H. Chen, Evaluation on Applicability of Reynolds Equation for Squared Transverse Roughness Compared to CFD, ASME Journal of Tribology, vol. 129, iss. 4, pp. 963-967, 2007, doi: $10.1115 / 1.2768619$

[28] Z. Song, F. Guo, Y. Liu, S. Hu, X. Liu, Y. Wang, Investigation of Slip/No-Slip Surface for TwoDimensional Large Tilting Pad Thrust Bearing, Industrial Lubrication and Tribology, vol. 69, no. 6, pp. 995-1004, 2017, doi: 10.1108/ILT-062017-0152

[29] ANSYS, ANSYS Fluent, version 16.0: user manual, ANSYS, Inc., Canonsburg, USA, 2017.

[30] J.H. Choo, R.P. Glovnea, A.K. Forrest, H.A. Spikes, $A$ Low Friction Bearing Based on Liquid Slip at the Wall, ASME Journal of Tribology, vol. 129, iss. 3, pp. 611-620, 2007, doi: 10.1115/1.2736704
[31] P. Zwart, A.G. Gerber, T. Belamri, A Two-Phase Flow Model for Predicting Cavitation Dynamics, in Proceedings of the fifth international conference on multiphase flow, Yoko-hama, Japan, May, 2004.

[32] Y. Hori, Hydrodynamic Lubrication, Tokyo: Springer-Verlag, 2006.

[33] B.J. Hamrock, S.R. Schmid, B.O. Jacobson, Fundamentals of Fluid Film Lubrication (Dekker Mechanical Engineering) $2^{\text {nd }}$ Edition, CRC Press, 2004.

[34] H. Zhang, M. Hua, G.N. Dong, D.Y. Zhang, K.S. Chin, Boundary Slip Surface Design for High Speed Water Lubricated Journal Bearings, Tribology International, vol. 79, pp. 32-41, 2014. doi: 10.1016/j.triboint.2014.05.022

[35] P.N. Shankar, M.D. Deshpande, Fluid Mechanics in the Driven Cavity, Annual Review of Fluid Mechanics, vol. 32, pp. 93-136, 2000, doi: 10.1146/annurev.fluid.32.1.93 